\title{
PRESENTACIÓN
}

\section{Resultados de serología de la Encuesta Nacional de Salud 2000}

D entro del cúmulo de información susceptible de ser recabada sistemáticamente sobre el estado de salud de la población es especialmente importante aquella proveniente de las encuestas serológicas, o bien del componente de muestreo biológico de encuestas de objetivo múltiple, como es el caso de la Encuesta Nacional de Salud 2000 (ENSA 2000). Este tipo de encuestas nos permiten complementar con parámetros biológicos específicos de diferentes marcadores de riesgo y enfermedades, la información que se reporta dentro de los sistemas de estadísticas rutinarias en el país. Dado que se trata de un esfuerzo con objetivos específicos y metodología común que se lleva a cabo en el territorio nacional, provee de información de gran veracidad e importancia para la toma de decisiones y la evaluación de programas y políticas nacionales.

Es importante, entonces, destacar que las mediciones realizadas en las muestras de suero de los participantes en esta encuesta nos permiten hacer un retrato certero y objetivo, tanto de la frecuencia y distribución de padecimientos infecciosos como de los diferentes factores de riesgo asociados con la morbilidad poblacional.

La ENSA 2000 constituye el tercer gran esfuerzo en México, y el más grande, para hacer un levantamiento sistemático de muestras serológicas de un sector representativo de la población nacional. El primero, llevado a cabo en 1974, se abocó a buscar anticuerpos para amibiasis, tifoidea, brucelosis, hepatitis A y B, sarampión, rubeola y parotiditis, entre otros padecimientos. Sin embargo, el muestreo no fue probabilístico ni se incluyó a población rural, por lo que era difícil hacer inferencias confiables respecto de la población en general.

Luego, poco después de la creación del Sistema de Encuestas Nacionales en Salud, se diseñó la Encuesta Nacional Seroepidemiológica, efectuada en 1987, la que se construyó con base en un muestreo probabilístico y en la que se recabaron alrededor de 70000 muestras, cuya representatividad y nivel de desagregación alcanzaba el ámbito estatal.
En comparación, para la ENSA 2000 se recolectaron más de 80000 muestras de suero de personas de todas las edades (excepto menores de un año) y más de 40000 muestras para identificación de DNA en adultos (mayores de 20 años). Estas muestras fueron concentradas en el laboratorio del banco de sueros y DNA del Instituto Nacional de Salud Pública (INSP) y pueden ser utilizadas mediante protocolos específicos de investigación.

La ENSA 2000 se basa en un muestreo probabilístico, polietápico, estratificado y por conglomerados, lo que permite aplicar con un alto grado de confiabilidad factores de expansión para lograr conclusiones sobre el estado de salud de la población en general. Además, es pertinente comentar que la metodología y los procedimientos utilizados en la encuesta mencionada fueron revisados y aprobada por la Comisión de Ética y Bioseguridad del INSP y que los participantes otorgaron consentimiento informado, incluyendo tanto la participación como la utilización subsecuente de las muestras biológicas con fines estadísticos y de investigación.

Los artículos del presente suplemento especial de Salud Pública de México aportan al cumplimiento de algunos de los objetivos enunciados por la ENSA 2000, como el caso de la estimación de la seroprevalencia de factores de riesgo y anticuerpos contra enfermedades infecciosas específicas, así como la evaluación de programas nacionales de salud, concretamente en lo relativo al Programa Nacional de Vacunación.

Así, Villalpando y colaboradores, al realizar el análisis de asociación entre las concentraciones séricas de glucosa, insulina y lípidos en una muestra de jóvenes entre 10 y 19 años, nos alertan sobre una fuerte asociación entre la obesidad juvenil y el riesgo de desarrollar algunas de las anormalidades del síndrome metabólico. Estos resultados son de gran relevancia, ya que nos obligan a redoblar esfuerzos en nuestros programas de acción dirigidos a la prevención de la obesidad infantil.

Por su parte, Olaiz-Fernández y colaboradores documentan que la diabetes mellitus continúa siendo 
un grave problema de salud en nuestro país, agravado en los grupos sociales de menor ingreso con dificultad para acceder al tratamiento. Los resultados de este grupo de autores también sugieren la necesidad de ampliar acciones hacia aquellos sujetos que padezcan hipertensión, dislipidemia, obesidad o microalbuminuria.

La obesidad es un problema creciente en nuestro país, asociada frecuentemente con otros trastornos, por lo que Barquera y colaboradores reportan que los adultos con obesidad tienen mayor probabilidad de tener hipertrigliceridemia e hipercolesterolemia que otros adultos con un índice de masa corporal normal, y que es cuatro veces más probable que desarrollen trastornos lipídicos, por lo que es necesario vigilar e intervenir en la creciente tendencia de la obesidad, ya que se asocia de manera importante con dislipidemias.

Otro de los factores de riesgo cardiovascular poco atendido es la concentración de proteína $C$ reactiva en el individuo. Flores y colaboradores concluyeron que existe una prevalencia alta de este factor en adultos mexicanos, a la que hay que poner especial atención, independientemente de otros factores que predisponen a trastornos cardiovasculares.

Un ejemplo de la utilidad de la serología para la evaluación de los programas de inmunización lo constituye el artículo de Ruiz-Gómez y colaboradores, que analiza la frecuencia y distribución de la prevalencia de anticuerpos contra la poliomielitis en niños menores de 10 años, y registra un porcentaje de 99.3 de seropositividad, en el que pese a lo positivo del índice, hay que atender el $0.7 \%$ restante, que en el ámbito nacional corresponde a cerca de 190 mil niños, que además se concentran geográficamente en la región sur del país.

En un segundo artículo, los mismos autores analizan los anticuerpos contra el sarampión en niños de 1 a 9 años. De sus resultados, por factores de expansión, se estima que unos 417000 niños en el ámbito nacional presentan títulos negativos de anticuerpos, es decir, son susceptibles de adquirir y transmitir el virus, por lo que pese a que la cobertura es adecuada y equitativa, sin diferencias apreciables por factores socioeconómicos, es necesario reforzarla, para proteger a esta población infantil y mantener al país libre de la circulación de sarampión.

La equidad reflejada por la cobertura de vacunación contra el sarampión contrasta con la distribución infecciosa de la hepatitis A. Debido a la no inclusión de esta vacuna en nuestro esquema de vacunación, debido a su dinámica de contagio, sigue respondiendo a factores de desigualdad social, al presentarse mayor seroprevalencia en el cuartil de menores ingresos de la población, sobre todo el que coincide con el entorno rural y la ausencia de servicios sanitarios. Es por ello que Valdespino y colaboradores sugieren valorar la posibilidad de introducir un esquema de vacunación para este padecimiento que, por otra parte, sufre una transición hacia grupos de edad más avanzada, grupo poblacional en los que este padecimiento puede tener complicaciones importantes. La información reportada sobre hepatitis A sin duda será un insumo importante para evaluar la posible inclusión de esta vacuna en el esquema nacional de vacunación.

Valdespino y colaboradores complementan el estudio de la hepatitis en México con dos artículos adicionales. El primero sobre la seroprevalencia de la hepatitis $C$ en adultos, donde identifican el padecimiento como un problema emergente de salud pública, que se agrava si se toma en cuenta que de 60 a $85 \%$ de los sujetos que la sufren desarrollan cronicidad, la cual puede complicarse con cirrosis hepática y cáncer primario de hígado. El segundo artículo se refiere a la prevalencia de la hepatitis $\mathrm{B}$ y el estado de portador crónico en adultos mexicanos, donde se destaca que, pese a que el patrón epidemiológico identificado es bajo respecto de los datos internacionales, el número de portadores crónicos puede resultar un problema dada la dinámica de la transición demográfica en el país.

La prevalencia de infección por VIH en la población general y factores relacionados fue estimada por García y colaboradores, siguiendo la recomendación diagnóstica de ONUSIDA, en una exploración que pudo además establecer una comparación entre etapas gracias a los datos de la Encuesta Nacional Seroepidemiológica de 1987. De entonces a ahora, la seroprevalencia en hombres adultos se ha incrementado 10 veces, y se observan datos de diseminación de la epidemia a grupos no considerados de riesgo inicialmente. Destaca en este trabajo la importancia del uso de drogas intravenosas como un factor de riesgo para la infección de $\mathrm{VIH}$ y nos alerta sobre la importancia de realizar intervenciones preventivas en este grupo poblacional.

Las enfermedades de transmisión sexual tienden a generar un subreporte de los casos, respecto de los que se podrían inferir mediante la cuantificación del antecedente infeccioso, como es el caso de la estimación de anticuerpos contra el treponema pallidum, causante de la sífilis, llevada a cabo por Conde-González y colaboradores, donde la diferencia entre uno y otro dato es considerable. Adicionalmente, los factores de riesgo de las enfermedades venéreas son complejos, pues pasan por aspectos sociodemográficos y de género, así como la persistencia de prácticas sexuales no seguras. Los autores identifican varias intervenciones posibles en el tema.

La ENSA 2000 incluyó una submuestra de adultos en ayuno, sobre la que Barquera y colaboradores informan los resultados comparativos con el resto de 
los encuestados, en los que no encontraron diferencias significativas que pudieran afectar los resultados de la medición bioquímica de riesgo cardiovascular efectuada sobre la submuestra.

Por último, el número cierra con el artículo de Sepúlveda y colaboradores, en el que se detallan el diseño conceptual y muestral y la metodología de la ENSA 2000, en cuya operación se obtuvieron las muestras serológicas utilizadas por los equipos de investigadores que publican sus resultados en este suplemento especial.

Salud Pública de México presenta de esta manera un compendio de estudios que, tomados en su conjunto, delinean un perfil epidemiológico que hay que atender de manera prioritaria y sugieren intervenciones que habrá que adoptar a corto y mediano plazo para acercarnos, como país, a un estadio de equidad inmunológica, sin menoscabo de atender paralelamente los rezagos sociales que acentúan muchos de estos padecimientos.

De esta forma se pone a disposición de los trabajadores de salud pública información de gran utilidad que permite la planeación en la orientación de las políticas de vacunación, aunque tenemos que establecer puntualmente que es importante encontrar en un futuro próximo los mecanismos para que información como ésta llegue oportunamente a los tomadores de decisiones.

Por último, es para mí un gran gusto reconocer que en este número de la revista también se rinde homenaje a un gran médico mexicano, al Dr. Jesús Kumate Rodríguez. Qué mejor manera de celebrar a un gran maestro que con excelentes trabajos de investigación llevados a cabo por muchos de sus alumnos. El Dr. Kumate ha sido un gran impulsor de la salud pública en nuestro país. Fue sin duda el arquitecto de las acciones que llevaron a la modernización del sistema de vigilancia epidemiológica y el impulsor del Programa Ampliado de Inmunizaciones, que ha llevado al país a contar con uno de los mejores y más completos esquemas de vacunación, que han contribuido, entre otros logros, a la eliminación del sarampión y de la poliomelitis en México.

Mauricio Hernández Ávila*

* Subsecreario de Prevención y Promoción de la Salud. Secretaría de Salud. México 